American Journal of Infectious Diseases 6 (2): 50-53, 2010

ISSN 1553-6203

(C) 2010 Science Publications

\title{
Comparison of Tuberculin Skin Test and Interferon Gamma Assay for the Diagnosis Latent Tuberculosis
}

\author{
Abolfazl Poorhasan, Mehdi Haghdoost and Omid Mashrabi \\ Department of Infectious Diseases, Faculty of Medicine, \\ Tabriz University of Medical Sciences, Tabriz, Iran
}

\begin{abstract}
Problem statement: After HIV infection, TB is the most common cause of mortality due to a single infectious agent. Until 1985, tuberculosis cases in the world were decreasing every year, but then with the prevalence of HIV infection, TB cases are increasing every year. Obviously, with this prevalence of tuberculosis, the best way to control it is to track the patient and identify the people with latent TB and treat them. One third of the world population is infected with latent tuberculosis micro bacterium and tuberculosis causes two million deaths a year. Identification of people with latent TB infection is the primary aim to eradicate $\mathrm{TB}$ in the community. The method to measure gamma interferon in the blood called QuantiFERON®-TB Test is a promising diagnostic test for diagnosing latent TB infection and is more beneficent than compared to PPD. The aim of this study is to compare IFN- $\delta$ test and PPD test in diagnosis of patients with latent TB. Approach: In a crosssectional descriptive-analytic study on 76 patients referred to the clinic or infectious diseases ward of Imam Reza or Sina hospitals or Tabriz University of Medical Sciences during 2007 and 2008, the patients whose CXR had fibrotic images or calcification compatible with latent TB infection were included in the study and the correlations between IFN- $\delta$ and PPD tests was evaluated. Results: Mean age of the patients in this study was $64.96 \pm 4.54$ years with minimum 43 and maximum 81 of age. About $33.3 \%$ of people under study had a positive PPD test and $4 \%$ of the patients had a positive IFN- $\delta$ and the correlation coefficient between IFN- $\delta$ test and PPD test was estimated at 0.376 $(\mathrm{p}<01 / 0)$. Age and gender had no effect on positivity of the results of PPD test and IFN$\delta$ tests. Conclusion: Capability of IFN- $\delta$ test in diagnosis of latent tuberculosis infection is comparable to that of PPD test; however, the correlation coefficient between the two tests was low and also, unlike other studies, gender and age had no effect on positivity or negativity of PPD and IFN- $\delta$ tests.
\end{abstract}

Key words: Purified derivative protein, green calamity bacillus vaccine, tuberculosis, chest x-ray

\section{INTRODUCTION}

Introduction and importance of the subject: After HIV infection, TB is the most common cause of mortality due to a single infectious agent. Until 1985, tuberculosis cases in the world were decreasing every year, but then with the prevalence of HIV infection, TB cases are increasing every year (Mandell et al., 2005). Obviously, with this prevalence of tuberculosis, the best way to control it is to track the patient and identify the people with latent TB and treat them (Mandell et al., 2005). One third of the world population is infected with latent tuberculosis micro bacterium and tuberculosis causes two million deaths a year (Mandell et al., 2005).

In some people, the relative risk of transformation of latent TB to active TB is very high; like people with one of the following conditions: diabetes, chronic renal failure, Gastrectomy, limb transplanting, head and neck carcinoma, pregnancy, receiving corticosteroids, malnutrition and HIV infected people (Mandell et al., 2005). All HIV-positive people with latent TB, if not treated, will eventually develop active TB (Mandell et al., 2005).

Regarding these facts, identification of people contaminated with tuberculosis Mycobacterium is necessary for TB control (Mandell et al., 2005). Since over than 30 years ago, treatment of latent TB infection to prevent active disease has been an essential part of TB control programs in developing countries (Mandell et al., 2005). Currently Tuberculin skin test, known as PPD test, is the Choice method for diagnosis of latent TB in the world.

Corresponding Author: Mashrabi Omid, Department of Infectious Diseases, Faculty of Medicine, Tabriz University of Medical Sciences, Tabriz, Iran Tel: +98-9144049694/+98-4226226106 
During recent years, more attention has been drawn to a new test called Quantiferon-TB Test in which the level of IFN- $\delta$ in blood is measured. This test is currently under study over the world and in some areas the correlations between IFN- $\delta$ and PPD test was high and in some other regions this correlation is low. The aim of this study is to compare IFN- $\delta$ and PPD test in diagnosis of patients with latent TB.

\section{MATERIALS AND METHODS}

In a cross-sectional descriptive-analytic study on 76 patients referred to the clinic or infectious diseases ward of Imam Reza or Sina hospitals or Tabriz University of Medical Sciences during 2007 and 2008, the patients whose CXR had fibrotic images or calcification compatible with latent TB infection were included in the study and the correlation between IFN- $\delta$ and PPD tests was evaluated.

Sample volume to be studied was estimated at 76 people based on the following formula:

$$
\mathrm{N}=\left[\left(\mathrm{Z}_{\mathrm{a}}+\mathrm{Z}_{\mathrm{B}}\right) \div\left(\mathrm{C}_{1}-\mathrm{C}_{2}\right)\right]^{2}+3
$$

Inclusion criteria: Patients in Infectious diseases wards of these two medical centers and the patients referred to clinics of infectious diseases who had fibrotic images and calcification compatible with latent TB in their CXR were included in this study (CXR was performed only on patients with graphic indication).

\section{Exclusion criteria:}

- People with positive Wright and Coombs Wright test results (gamma interferon level at Brucellosis rises, so brucellosis tests were performed on all subjects before inclusion in the study and the cases with positive results were excluded)

- People not consent for PPD test and blood sampling for IFN- $\delta$

- People with a history of TB treatment

- Patients with Ralper clinical symptoms were also excluded

After selecting patients, the PPD test was performed with the following conditions: To perform this test, firstly, area of the left anterior forearm was washed with alcohol and then cleaned with distilled water to clear the skin of alcohol because remaining alcohol on the skin will lead to false negative test result due to denaturation of proteins in PPD solution. After drying the skin, 5 units of $0.1 \mathrm{~mL}$ PPD solution was injected intraperitoneally and the test results were read after 48$72 \mathrm{~h}$. To read the PPD test result, the indurations perpendicular to the injection line should be measured and notes in terms of millimeters. Then $1 \mathrm{cc}$ of complete blood was taken from the subjects and after the blood clots inside the test tube, it was centrifuged for 5 min until serum was separated, then the serum over the clot was drawn into plastic tubes and freeze in $-70^{\circ} \mathrm{C}$ temperature. After completion of this part and preparing 76 samples, the serums were transferred to the laboratory of Pharmaceutical Applied Researches Center and the amount of their IFN- $\delta$ were measured by Elisa method. Based on previous studies, positive Cutoff point of this test was assumed at $10 \mathrm{pgr} \mathrm{mL} \mathrm{m}^{-1}$; so, the samples with $10 \mathrm{pgr} \mathrm{mL}^{-1} \leq \mathrm{IFN}-\delta$ were considered positive and the samples with IFN- $\delta$ less than this amount were considered negative (Brock et al., 2004).

Coombs Wright and Wight tests were also performed on all the patients under study and patients with positive test result were excluded from the study. Written consent of the patients was obtained for PPD test and taking blood sample for IFN- $\delta$ test and non-consent cases were excluded.

Data obtained through SPSS statistical software (Ver. 13) and Pearson Correlation statistical tests were statistically analyzed. In this study, $p<0.05$ has been considered as the significant level.

\section{RESULTS}

Mean age of patients in this study was $64.96 \pm 4.54$ in the range of $43-81$ years. About 50 cases $(66.6 \%)$ of the patients under study were male and 26 cases $(33.4 \%)$ were female. All of the cases had the history of BCG vaccination during childhood. PPD test result in 25 patients, 15 males (60\%) and 10 females $(40 \%)$, was positive (PPD $\geq 10 \mathrm{~mm})$. Average age of the patients with positive PPD was $62.54 \pm 2.66$ years. IFN$\delta$ test was positive only in 3 patients $(2$ males and 1 female) whose PPD test result were also positive and over $15 \mathrm{~mm}$. Mean age of the patients with positive IFN- $\delta$ test result was $61.84 \pm 3.43$ years. PPD test results in patients were shown in Fig. 1.

In order to evaluate the correlation coefficient between IFN- $\delta$ and PPD tests, Pearson correlation test was applied. The correlation coefficient between IFN- $\delta$ and PPD tests on the cases under study was 0.376 and significant $(\mathrm{p}<0.01)$.

Positivity rate of PPD test $(p=0.509)$ and IFN- $\delta$ test $(\mathrm{p}=0.413)$ had no significant relationship with the age of the patients. Positivity rate of PPD test $(\mathrm{p}=0.131)$ and IFN- $\delta$ test $(\mathrm{p}=0.338)$ had no significant relationship with the gender of the patients. 
Am. J. Infect. Dis., 6 (2): 50-53, 2010

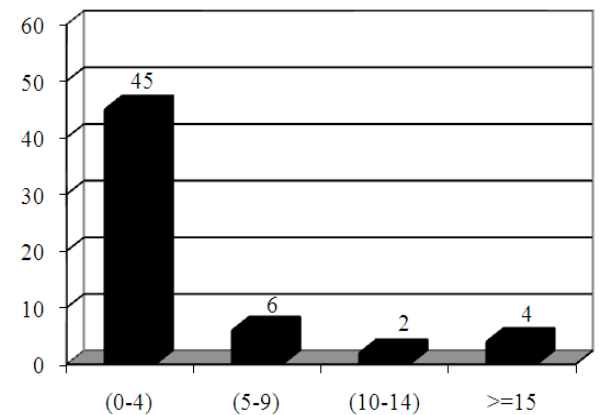

Fig. 1: PPD test results in patients under study

\section{DISCUSSION}

Due to many cases of false positive and negative PPD test results, this test cannot be used as the gold standard and with the re-prevalence of TB infection along with the growth of HIV infection, lack of such tests for diagnosing latent TB infection are more obvious; since the best way to control TB in the community is to identify and treat people with latent TB. PPD test is a simple and inexpensive test with medium to high sensitivity, which depends on population screening, but its specificity is low (Huebner et al., 1993). Many studies indicate a positive relationship between positive PPD test and the risk of prospective active TB (Huebner et al., 1993; Ewer et al., 2003; Mazurek et al., 2001). And several studies have shown that treatment of latent tuberculosis diagnosed by PPD test reduces active TB risk $60-90 \%$ (Brock et al., 2004).

But it needs a skilled tester, the booster phenomenon occurs and BCG vaccine and infection by non-TB Mycobacterium cause false positive test result.

IFN- $\boldsymbol{\delta}$ test: Have higher specificity than PPD test and no cross-reactivity with BCG vaccine or infection with other non-tuberculosis mycobacterium (Ewer et al., 2003; Brock et al., 2004; Mazurek and Villarino, 2003; Mori et al., 2004). It is fast operative and individualindependent (Mazurek et al., 2001; Lalvani, 2003; Pai et al., 2004; Chadha, 2003; Chadha et al., 2005; Alonso-Echanove et al., 2001). However, IFN- $\delta$ method is not assessed carefully enough and especially in children and immunosuppressive people and in countries where TB is endemic, it has not been yet accurately evaluated (Pai et al., 2004). In poor countries, PPD test is still widely in use as a valuable test. In India, PPD test has significant use for diagnosis of latent tuberculosis infection in children (Chadha, 2001).

On the other hand, as seen in epidemiologic studies, application of tests such as IFN- $\delta$ test on high risk people who must be tested annually would be more difficult (Chadha, 2001; 2003; Chadha et al., 2005). Many studies have suggested that the positive PPD test is associated with the risk of prospective active TB (Chadha, 2001; 2003; Brock et al., 2004).

To prove the relationship between positive IFN- $\delta$ test and the risk of prospective active TB, long-term cohort studies are needed. In such situation, IFN- $\delta$ test can be a good alternative for PPD test. In many studies, PPD test and IFN- $\delta$ test have been compared (Ewer et al., 2003; Brock et al., 2004; Mazurek et al., 2001; Pai et al., 2004) and in many of these studies the correlation coefficient between the two tests is high. In some studies, but the correlation coefficient between the two tests was low (Brock et al., 2004). In a study conducted in Ethiopia, the correlation coefficient between the two tests has been very low, while it has been higher in the study carried out in America (K>0.75) (Mandell et al., 2005).

In our study, the correlation coefficient between the two tests was positive and significant but lower than that in other studies $(\mathrm{K}<0.4)$ and IFN- $\delta$ test was positive only on three cases, but the notable fact in this study is that IFN- $\delta$ test was positive on cases where PPD test was also positive with a high and significant value. This may imply that if IFN- $\delta$ test is positive on a person, he can be said with certainty to be infected with latent TB; because in this study, in cases with Positive IFN- $\delta$ test, $15 \mathrm{~mm} \leq$ PPD, that this PPD size in all individuals with any underlying disease and in any population, has the treatment indication of tuberculosis; therefore, if this fact proven in other studies, it can be concluded that wherever IFN- $\delta$ test is positive (unlike different PPD sizes which should be interpreted based on age, place of living and underlying disease of the individual and determine the treatment indication) person will unconditionally have latent TB treatment indication.

Of course, PPD test may also be negative on TB infection, but 2 weeks after starting anti-TB treatment, PPD test will be positive (Brock et al., 2004). But IFN- $\delta$ test is negative up to one year after starting TB treatment (Mori et al., 2004; AlonsoEchanove et al., 2001).

Like our study, several studies have been conducted on this case around the world and the same results are observed in various studies that contradictory results can be seen in this case too. Even though the FDA test is approved, but due to these different results, researchers recently believe that some other new tests should replace IFN- $\delta$ and PPD tests. One of the new tests under much attention in recent years is IL-4 test (Brock et al., 2004; Mori et al., 2004).

IL-4 test is based on measuring the amount of interleukin-4 in the blood. This test is currently being 
probed and few surveys have been conducted on it, but based on these few studies, it has shown high test sensitivity and fewer false negative cases and it can be used for tracking after treatment of pulmonary tuberculosis (Ewer et al., 2003; Alonso-Echanove et al., 2001).

However, like the IFN- $\delta$ test, this test should also be evaluated in countries with endemic TB and investigated on people with latent TB and pulmonary TB.

In a study carried out recently in India, IFN- $\delta$ and IL4 tests were used simultaneously to identify latent tuberculosis that has shown better results rather than using each test alone. It is expected that such researches would be performed in our country in the future.

Considering the fact that Iran is among countries with endemic TB and the increase of IVDU and HIVpositive people and re-prevalence of TB, many researches, including gamma interferon and interleukin4 should be conducted in our country for the diagnosis of latent TB; so that these tests could be discussed on.

In this study, there was no relationship between age and sex and positivity of PPD and IFN- $\delta$ tests; While age was considered as a risk factor in some studies and positive PPD and IFN- $\delta$ cases has been increasing with increase of age (Ewer et al., 2003; Alonso-Echanove et al., 2001).

\section{CONCLUSION}

Correlation coefficient between the PPD test and gamma interferon was $r=0.376$. Age and sex had no effect on positivity of PPD and IFN- $\delta$ tests. IFN- $\delta$ test was positive wherever PPD size $\geq 15 \mathrm{~mm}$ which could indicate latent tuberculosis infection in any individual and any population.

Suggestions: It seems that yet some other studies are needed for replacing the IFN- $\delta$ test instead of PPD test; especially, studies should also be done on children and immunosuppressive people. Moreover, in order to state the association of positive IFN- $\delta$ test with prospective active TB risk, long-term cohort studies should be performed. However, what was expressed in this study like other studies was that PPD test is currently the only available applicable test to diagnose latent TB infection and replacement of IFN- $\delta$ test requires further studies; because, Iran is among endemic countries in terms of TB and, as observed, the correlation coefficient between the two tests was low in such country.

\section{REFERENCES}

Alonso-Echanove, J., R.M. Granich, A. Laszlo, G. Chu and N. Borja et al., 2001. Occupational transmission of Mycobacterium tuberculosis to health care workers in a university hospital in Lima, Peru. Clin. Infect. Dis., 33: 589-596. PMID: 11477527
Brock, I., K. Weldingh, T. Lillebaek, F. Follmann and P. Andersen, 2004. Comparison of tuberculin skin test and new specific blood test in tuberculosis contacts. Am. J. Respir. Crit. Care Med., 170: 65-69. PMID: 15087297

Chadha, V.K., 2001. Tuberculin test. Indian J. Pediatr., 68: 53-58. PMID: 11237237

Chadha, V.K., 2003. Epidemiological situation of tuberculosis in India. J. Indian Med. Assoc., 101: 144-147. PMID: 14603957

Chadha, V.K., P. Colford, P.S. Jagannatha, P.S. Vaidyanathan and K.P. Unnikrishnan, 2005. Average annual risk of tuberculous infection in India. Int. J. Tuberc. Lung Dis., 9: 116-118. PMID: 15675560

Ewer, K., J. Deeks, L. Alvarez, G. Bryant and S. Waller, 2003. Comparison of T-cell-based assay with tuberculin skin test for diagnosis of Mycobacterium tuberculosis infection in a school tuberculosis outbreak. Lancet, 361: 1168-1173. PMID: 12686038

Huebner, R.E., M.F. Schein and J.B. Bass Jr., 1993. The tuberculin skin test. Clin. Infect. Dis., 17: 968-975. PMID: 8110954

Lalvani, A., 2003. Spotting latent infection: the path to better tuberculosis control. Thorax, 58: 916-918. PMID: 14586040

Mandell, G.L., J.E. Bennett and R. Dolin, 2005. Principles Practice Infectious Diseases. 6th Edn., Churchill Livingstone, USA., ISBN: 13: 9780443068393,pp: 4000.

Mazurek, G.H. and M.E. Villarino, 2003. Guidelines for using the QuantiFERON-TB test for diagnosing latent Mycobacterium tuberculosis infection. MMWR Recomm. Rep., 52: 15-18. PMID: 12583541

Mazurek, G.H., P.A. LoBue, C.L. Daley, J. Bernardo and A.A. Lardizabal, 2001. Comparison of a whole-blood interferon gamma assay with tuberculin skin testing for detecting latent Mycobacterium tuberculosis infection. J. Am. Med. Assoc., 286: 1740-1747. PMID: 11594899

Mori, T., M. Sakatani, F. Yamagishi, T. Takashima and Y. Kawabe et al., 2004. Specific detection of tuberculosis infection: an interferon-gamma-based assay using new antigens. Am. J. Respir. Crit. Care Med., 170: 59-64. PMID: 15059788

Pai, M., L.W. Riley and J.M. Colford Jr., 2004. Interferon-gamma assays in the immunodiagnosis of tuberculosis: A systematic review. Lancet Infect. Dis., 4: 761-776. PMID: 15567126 